\title{
Pretraining Prevents Spatial Learning Impairment after Saturation of Hippocampal Long-Term Potentiation
}

\author{
Mona Kolstø Otnæss, Vegard Heimly Brun, May-Britt Moser, and Edvard I. Moser \\ Department of Psychology, Norwegian University of Science and Technology, 7491 Trondheim, Norway
}

Spatial learning is impaired by NMDA receptor antagonists at doses that block hippocampal long-term potentiation (LTP). The deficit is not observed in animals that have received spatial or nonspatial pretraining in a different water maze. To determine whether this conditional impairment reflects debilitating sensorimotor effects of NMDA receptor antagonists in naïve animals, we compared spatial learning in naïve and pretrained animals in which induction of LTP was blocked by a saturation procedure with no obvious effects on sensorimotor functions. Rats with unilateral hippocampal lesions were implanted with multiple bipolar stimulation electrodes in the angular bundle and a recording electrode in the dentate gyrus of the intact hemisphere. Half of the rats were pretrained to find a hidden platform in a water maze. A week later, pretrained and naïve rats received either high-frequency (HF) or low-frequency (LF) stim- ulation at $2 \mathrm{hr}$ intervals, until no further LTP could be induced. The stimulation did not interefere with performance on a balance task or a visual platform task. After stimulation, all rats were trained in a second water maze. Whereas naïve HF animals were impaired, pretrained HF animals acquired the new task rapidly and searched as extensively around the platform as LF control animals. These results suggest that pretraining prevents disruption of spatial learning after saturation of LTP in the absence of sensorimotor impairment, that hippocampal LTP might not be crucial for spatial representation per se, and that LTP may be involved only when spatial and contextual or procedural learning take place simultaneously.

Key words: spatial memory; hippocampus; LTP; memory; synaptic plasticity; water maze; rat
Long-term potentiation (LTP) is currently the main model for how memories are formed and stored in the hippocampal formation (Bliss and Collingridge, 1993). Much of the validity of the model rests on the apparently overlapping mechanisms of hippocampal LTP and hippocampus-dependent spatial learning, such as the need for NMDA receptor activation. NMDA receptor dysfunction impairs both hippocampal LTP and spatial learning (Morris et al., 1986; Sakimura et al., 1995; Tsien et al., 1996), as does interference with several other elements of the molecular cascade leading to LTP (e.g., Silva et al., 1992). Spatial learning is disrupted if LTP is "saturated" by repeated induction in a large number of synapses (McNaughton et al., 1986; Castro et al., 1989; Moser et al., 1998). Altogether, these data suggest that LTP-like enhancement of hippocampal synaptic transmission is necessary for spatial memory formation.

However, animals that have received spatial or nonspatial pretraining can learn a new water maze task even if LTP is blocked by NMDA receptor antagonists (Bannerman et al., 1995; Saucier and Cain, 1995). A similar effect of pretraining is not seen after hippocampal lesions (Bannerman et al., 1995). These observations raise the possibility that disruption of spatial learning after interference with LTP reflects impaired capacity of the hippocampus to perform processes other than those requiring LTPlike modifications.

There are at least two main interpretations of the effect of

Received Sept. 7, 1999; revised Oct. 18, 1999; accepted Oct. 19, 1999.

This work was supported by Norwegian Research Council Grants 115013/310, 115015/310, and 122512/310, Torstein Erbo's Foundation, the EWS Foundation, and Dr. Dedichen's Institute for Psychiatric Research. We are grateful to A. K. Amundgård, K. Barmen, and K. Haugen for technical assistance.

Correspondence should be addressed to Mona Kolst $\varnothing$ Otnæss at the above address. E-mail: monao@svt.ntnu.no.

Copyright (C) 1999 Society for Neuroscience $\quad 0270-6474 / 99 / 190001-\bullet \$ 05.00 / 0$ pretraining. On one hand, impaired spatial learning in naïve rats could reflect the debilitating effects of the antagonists on the acquisition of basic sensorimotor strategies required for successful performance in a water maze (Saucier and Cain, 1995; Cain et al., 1996). On the other hand, NMDA receptor-dependent LTP might be necessary for learning of only a subset of the behavioral components of the water maze task, e.g., general search strategies crucial for any water maze task (Bannerman et al., 1995). Both hypotheses assume that the critical LTP-dependent process was already completed by the time the animals are trained under NMDA receptor blockade, but only the latter leaves a role for LTP in some component of spatial learning. To resolve the issue, we asked whether pretraining rescues learning when LTP is blocked without accompanying sensorimotor dysfunctions. We saturated LTP in the perforant path synapses of the dentate gyrus and tested whether spatial learning took place in pretrained animals.

\section{MATERIALS AND METHODS}

Subjects. Thirty-eight male Long-Evans rats (250-400 gm; M\&B) were housed in groups of four to six in large transparent polycarbonate cages

This article is published in The Journal of Neuroscience, Rapid Communications Section, which publishes brief, peerreviewed papers online, not in print. Rapid Communications are posted online approximately one month earlier than they would appear if printed. They are listed in the Table of Contents of the next open issue of JNeurosci. Cite this article as: JNeurosci, 1999, 19:RC49 (1-5). The publication date is the date of posting online at www.jneurosci.org.

http://www.jneurosci.org/cgi/content/full/3796 
$(55 \times 45 \times 35 \mathrm{~cm})$ with food and water available ad libitum. The animals were kept on a $12 \mathrm{hr}$ light/dark schedule and tested in the dark phase.

Surgery. Rats anesthetized with Equithesin received unilateral lesions as described previously (Moser and Moser, 1998). Two weeks later, electrodes were implanted (Moser et al., 1998). Briefly, three bipolar SNEX100 stimulation electrodes (Rhodes Medical Instruments, Woodland Hills, CA) were implanted in the medial, middle, and lateral angular bundle of the intact hemisphere $0.1,0.2$, and $0.3 \mathrm{~mm}$ anterior and 3.0, 4.0, and $5.0 \mathrm{~mm}$ lateral to lambda, respectively. Two recording electrodes (twisted $70 \mu \mathrm{m}$ Teflon-insulated stainless steel wires; Goodfellow, Cambridge, UK) were implanted in the granule cell layer of the ipsilateral dentate gyrus, $3.5 \mathrm{~mm}$ posterior and $2.4 \mathrm{~mm}$ lateral to bregma, with depth determined on the basis of field potentials in response to $0.2 \mathrm{~Hz}$ stimulation through the middle stimulation electrode. Electrodes, two ground screws, a return screw for monopolar stimulation, and four anchoring screws were cemented to the skull using dental acrylic.

Spatial pretraining. Two weeks later, 19 rats were trained to find a hidden platform in opaque water in a Morris water maze $(198 \mathrm{~cm}$ diameter; $50 \mathrm{~cm}$ depth; water depth, $40 \mathrm{~cm}$; temperature, $\left.25 \pm 1{ }^{\circ} \mathrm{C}\right)$. The pool had four platforms $(10 \mathrm{~cm}$ diameter $)$ that could be regulated between an available level and an unavailable level (1.5 and $22 \mathrm{~cm}$ below the surface, respectively). The training room was $4 \times 6 \mathrm{~m}^{2}$. Each rat was trained for $5 \mathrm{~d}$ ( 4 trials morning and 4 trials evening) to find one platform at a fixed position (NE, NW, SE, or SW). Start positions alternated between N, S, E, and W in a pseudo-random fashion. Maximum trial length was $120 \mathrm{sec}$. The rat was left on the platform for $30 \mathrm{sec}$ after each trial. Trials 17 and 33 served as probe tests with the platform in its lower, unavailable position for $60 \mathrm{sec}$, before it was raised to the upper position so the rat could climb onto it. The swim pattern of each rat was identified and stored by a tracking system (Moser and Moser, 1998).

Stimulation procedure. Pretrained rats were matched with respect to time spent in the platform zone on the last spatial probe test and assigned to a high-frequency (HF) stimulated experimental group or a lowfrequency (LF) control group. After pretraining, all rats received extensive handling and habituation to the test boxes.

Seven days after pretraining, evoked field potentials were recorded at $20 \mathrm{kHz}$ from one electrode in the dentate gyrus during seven recording sessions at $2 \mathrm{hr}$ intervals. Between sessions, the rats rested in the animal room $4 \mathrm{~m}$ away. Each session consisted of $18100-\mu$ sec pulses delivered at $0.2 \mathrm{~Hz}$ through the middle stimulation electrode at three pulse intensities (population spike threshold, $\sim 1 \mathrm{mV}$ population spike, $\sim 3 \mathrm{mV}$ population spike; $50-1000 \mu \mathrm{A})$. Stimulation intensities were not different between naïve and pretrained animals (e.g., $201 \pm 34$ and $232 \pm 42 \mu \mathrm{A}$, respectively, at spike threshold; means \pm SEM).

Immediately after the third, fourth, fifth, and sixth recording (at 5, 7, 9, and $11 \mathrm{hr}$ ), the rats received either LF or HF stimulation. HF stimulation consisted of eight trains of eight pulses at $400 \mathrm{~Hz}$ with a $2 \mathrm{sec}$ intertrain interval (pulse width, $100 \mu \mathrm{sec}$ ); LF stimulation consisted of eight single pulses at $0.5 \mathrm{~Hz}$. The current was passed systematically between pairs of six stimulation sites defined by the medial $(a, b)$ and lateral $(c, d)$ stimulation electrodes, the tip of the middle stimulation electrode deep in the angular bundle $(e)$, and the return screw on the skull above and caudal to the stimulation electrodes $(f)$ (Fig. 1). Stimulation intensities were equal for HF and LF animals. For the first 21 rats, we stimulated at $1500 \mu \mathrm{A}$ when anode and cathode were at different sides of the angular bundle and at $800 \mu \mathrm{A}$ when the poles were on the same side of the bundle. For the following 17 rats, the intensity was increased to 2000 and $1500 \mu \mathrm{A}$, respectively, to increase the proportion of animals reaching our saturation criterion (see below). The two sets of tetanization intensities were equally distributed across groups and were not associated with different behavioral results.

New learning after stimulation. Three hours after tetanization, the animals were trained in a new water maze $(200 \mathrm{~cm}$ diameter, $50 \mathrm{~cm}$ depth, opaque water at $40 \mathrm{~cm}$ depth, $25 \pm 1^{\circ} \mathrm{C}$ ) in a new room with unique spatial layout, cue configuration, and size $\left(3 \times 4 \mathrm{~m}^{2}\right)$. All rats, pretrained and naïve, were trained to find a fixed platform in the new maze. Rats were randomly assigned to one of the four platform positions. The training consisted of two trials per session (interval, $30 \mathrm{sec}$ ). Sessions were spaced by $\sim 1 \mathrm{hr}$. Pretrained rats received eight training sessions, with spatial probe tests (platform raised at $60 \mathrm{sec}$ ) before training and on sessions 4 and 8 . The naïve groups received 12 training sessions, with probe tests on sessions 4,8 , and 12 . In addition, time in the platform zone was calculated from the first $60 \mathrm{sec}$ of swimming on session 1 in these rats. When the escape latency was $<60 \mathrm{sec}$ ( 5 of 13 rats), zone times were expressed as percentages of total swim time on this test.

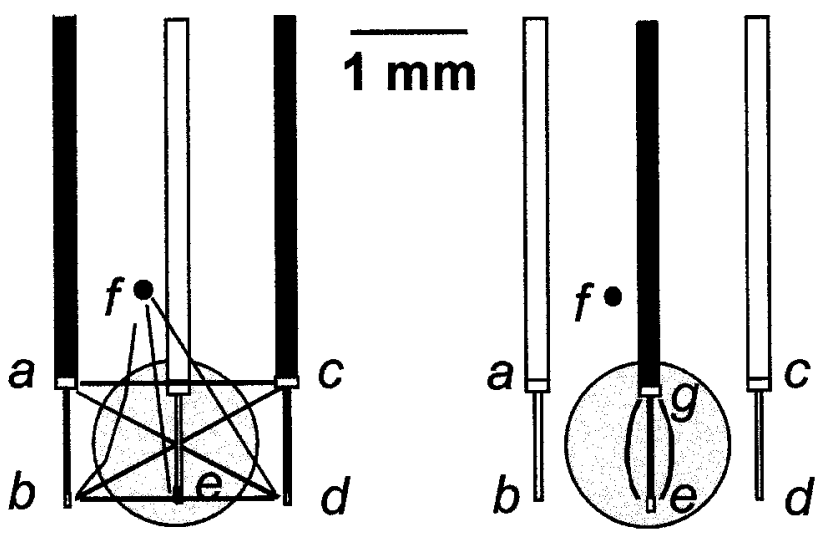

Figure 1. Stimulation protocols. Left, Induction of LTP by tetanic stimulation across the angular bundle (circle), with anode and cathode varied between six stimulation sites $(a-f)$. Sequence of stimulation: at 5 and $9 \mathrm{hr}$, $e f, a d, b c, a c, b d, a b, c d$; at 7 and $11 \mathrm{hr}, b f, d f, d a, c b, c a, d b, b a, d c$. For each combination, eight trains (high-frequency group) or eight single pulses (low-frequency group) were delivered, with combinations separated by 30 sec intervals. Right, Estimate of residual LTP by tetanization through the central electrode only $(g, e)$.

Sensorimotor tests. Two tests were designed to evaluate sensorimotor abilities. The first was a balance test in which a 3.5 -cm-wide wooden plank was placed $10 \mathrm{~cm}$ over the surface of the water maze. The rats, all naive to the task, were placed on the middle and were required to balance to one of the end points (1.0 m away). On the second test, the rats swam to and climbed an elevated platform ( $2 \mathrm{~cm}$ above the surface) in the water maze. White curtains were drawn around the pool. Latency to reach and climb onto the platform was recorded. The procedure was repeated four times at $1 \mathrm{~min}$ intervals and with different start positions.

Estimate of residual LTP. Two hours after the last swim trial, field potentials were recorded as above (session 8 at $29 \mathrm{hr}$ ), and HF stimulation was subsequently delivered at $1000 \mu \mathrm{A}$ between the naïve cathode and the anode of the middle electrode in all rats (Fig. 1g,e). HF stimulation consisted of eight trains $(0.5 \mathrm{~Hz})$ of eight pulses $(400 \mathrm{~Hz})$ repeated twice at the same polarity (60 sec interval). Field potentials were recorded again $1 \mathrm{hr}$ later to determine residual LTP (session 9). HF rats with $>10 \%$ increase of the EPSP slope on this test (four naive and two pretrained) were excluded from further analysis.

Histology. The rats received an overdose of Equithesin and were perfused intracardially with saline and $4 \%$ formaldehyde. Preparation of brain sections and calculation of volume of residual hippocampal tissue have been described previously (Moser and Moser, 1998).

\section{RESULTS}

\section{Histology}

All rats had damage to one hippocampus as intended, whereas the implanted contralateral hippocampus was intact except for the electrode traces. On the lesioned side, only small remnants of hippocampal tissue were left at the septal and temporal poles (HF pretrained, $8.7 \pm 1.9 \%$; LF pretrained, $8.7 \pm 1.9 \%$; HF naïve, $8.8 \pm 2.9 \%$; LF naïve, $12.9 \pm 2.5 \%$; means \pm SEM). ANOVA showed no significant effects on residual tissue of stimulation (HF or LF), training (pretrained or naïve), or stimulation $\times$ training (all $F<1$ ).

\section{Pretraining}

During the 10 pretraining sessions, all rats learned to swim directly to the hidden platform. Mean latency \pm SEM to find the platform was $91.7 \pm 5.2 \mathrm{sec}$ on session 1 and $10.4 \pm 1.2 \mathrm{sec}$ on session 10 , with means consistently $<20 \mathrm{sec}$ during the second half of training. On the final spatial probe test, all rats searched around the platform $(31.0 \pm 3.6 \%$ of total search time within a 64-cm-diameter circle around the platform; value expected by 


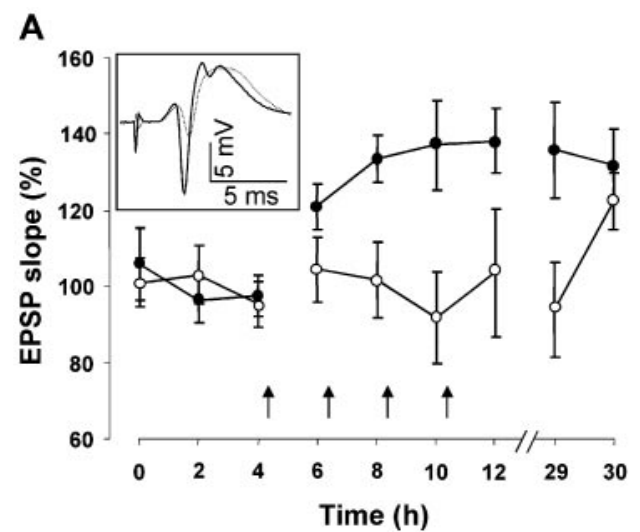

Figure 2. Potentiation of field potentials in perforant path synapses of the dentate gyrus. Normalized values for EPSP slope (relative to baseline sessions) are given for rats receiving $\mathrm{HF}$ or $\mathrm{LF}$ stimulation (means $\pm \mathrm{SEM}$ ). HF or LF stimulation (arrows) was delivered immediately after recording at 5, 7, 9, and $11 \mathrm{hr}$. The animals were trained in the water maze at 14-26 hr. Residual LTP was estimated by tetanic stimulation through the central electrode at 29 hr (both groups). $A$, Naïve rats; $B$, pretrained rats. Inset, Representative evoked potentials at $5 \mathrm{hr}$ (broken line) and $13 \mathrm{hr}$ (solid line). chance, $10.2 \%)$. There were no significant effects of group or group $\times$ session $($ all $F<1$ ). There was a significant main effect of zone on probe test times $\left(F_{(3,45)}=31.6 ; p<0.001\right)$ and a significant effect of session on the escape latencies $\left(F_{(7,105)}=55.8\right.$; $p<0.001)$.

\section{Stimulation and recording of evoked potentials}

Tetanic stimulation started after session 3 (at $5 \mathrm{hr}$ ). Animals in both the pretrained and the naïve HF groups showed a rise in EPSP slope compared with baseline from session 4 to session 7 (Fig. 2; 6-12 hr). The EPSP slope of the two LF groups remained at baseline level. There was a significant effect of stimulation (HF vs LF) on field EPSP slope $\left(F_{(1,16)}=9.7 ; p<0.01\right.$; sessions $\left.4-7\right)$, but no effect of training (pretrained vs naïve) or stimulation $\times$ training $(F<1)$. HF stimulation also lead to some increase in the population spike amplitude $(0.4 \mathrm{mV}$ at the high intensity), but this effect was present also in the LF group $(0.3 \mathrm{mV})$. The general increase in spike amplitude could reflect decreased brain temperature as the animals got habituated to the stimulation procedure during the course of the experiment (Moser et al., 1993).

\section{New learning}

None of the pretrained groups showed any spatial bias on the first trial in the new water maze $(60 \mathrm{sec}$ probe test; time in the platform zone: HF, $8.0 \pm 2.0 \%$; LF, $11.9 \pm 2.3 \%$; value expected by chance, $10.2 \%$ ).

\section{Pretrained animals}

Both pretrained groups (HF and LF) started out with lower escape latencies than the naïve groups, suggesting that they benefited from the pretraining (Fig. $3 A$ ). Both groups also quickly learned to swim directly to the platform (session effect, $F_{(5,75)}=$ $6.1 ; p<0.001$; group and session $\times$ group effects, $F<1$ ). Spatial probe tests conducted 0,4 , and $8 \mathrm{hr}$ after the start of training showed that both groups developed a clear preference for the platform region (Fig. $3 B$ ) and that their rate of learning was similar (Fig. $3 C$ ). ANOVA of swim times across pool quadrants revealed a significant effect of zone at 4 and $8 \mathrm{hr}\left(F_{(3,45)} \geq 14.6\right.$; $p<0.001)$ but with no zone $\times$ group interactions $(F<1)$. There was no group effect on the rate at which time in the platform zone increased during training $(F<1)$.

\section{Nä̈ve animals}

In the naïve animals, the initially high escape latencies were clearly reduced during training after both $\mathrm{HF}$ and LF stimulation (session, $F_{(8,88)}=9.8 ; p<0.001$; Figure $\left.3 D\right)$. Although stimulation condition had no effect on the escape latencies $(F<1)$, the
HF group was impaired on the spatial probe tests (Fig. 3E,F). ANOVA of swim times on the final test revealed significant effects of zone $\left(F_{(3,33)}=14.4 ; p<0.001\right)$ and zone $\times$ group $\left(F_{(3,33)}=3.1 ; p<0.05\right)$. Time in the platform zone increased faster in LF animals than HF animals $\left(F_{(3,33)}=2.9 ; p=0.05\right.$; Figure $3 F$ ).

Because the pretrained HF group developed slightly less LTP than in the naïve HF group (Fig. 2), we tested new learning in another set of animals (13 HF and $12 \mathrm{LF}$ ) with an identical pretraining history but more EPSP potentiation $(30.1 \pm 14.0 \%)$. Again, new learning was equally efficient in the HF and LF groups. Times in the platform zone on the probe test (trial 7) were $29.2 \pm 2.5$ and $31.0 \pm 4.4 \%$, respectively.

\section{Sensorimotor tests}

All rats balanced successfully across the elevated plank and climbed the raised platform in the water maze within few seconds $\left(\mathrm{HF}, 6.8 \pm 0.9 \mathrm{sec}\right.$; LF, $5.5 \pm 0.5 \mathrm{sec} ; t_{(25)}=1.4$; NS). Time to climb the platform did not correlate with time in the platform zone on the final probe trial $\left(r_{(25)}=0.1\right.$; NS).

\section{Residual LTP}

To estimate residual capacity for LTP, we tetanized between electrode poles never used in combination before, the tip and the shaft of the middle electrode. One hour later, there was no enhancement but rather a small decrease in the EPSP slope in the $\mathrm{HF}$ groups (pretrained, $-6.0 \pm 3.2 \%$; naïve, $-1.8 \pm 2.5 \%$; Fig. $2 B, C, 29$ vs $30 \mathrm{hr}$ ). Both LF groups showed a marked enhancement (pretrained, $33.0 \pm 8.7 \%$; naïve, $20.9 \pm 10.1 \%$ ). An ANOVA of EPSP slope values showed a significant effect of stimulation $\left(\mathrm{HF}\right.$ vs LF; $\left.F_{(1,19)}=20.6 ; p<0.001\right)$ but not of training (pretrained vs naïve; $(F<1)$ or stimulation $\times$ training $\left(F_{(1,19)}=2.7\right.$; NS $)$.

There was also a differential enhancement of the population spike amplitude (pretrained LF, $0.62 \pm 0.33 \mathrm{mV}$; naïve $\mathrm{LF}$, $0.73 \pm 0.31 \mathrm{mV}$; pretrained $\mathrm{HF}, 0.29 \pm 0.15 \mathrm{mV}$; naïve $\mathrm{HF}$, $-0.08 \pm 0.14 \mathrm{mV}$; stimulation, $F_{(1,20)}=5.6 ; p<0.05$; no effect of training, no interaction).

\section{DISCUSSION}

The main finding was that pretraining prevents the impairment of spatial learning after saturation of LTP in the perforant path synapses of the hippocampus. This is consistent with previous studies demonstrating intact spatial learning in pretrained animals when NMDA receptor-dependent LTP is blocked (Bannerman et al., 1995; Saucier and Cain, 1995). Rats treated with 

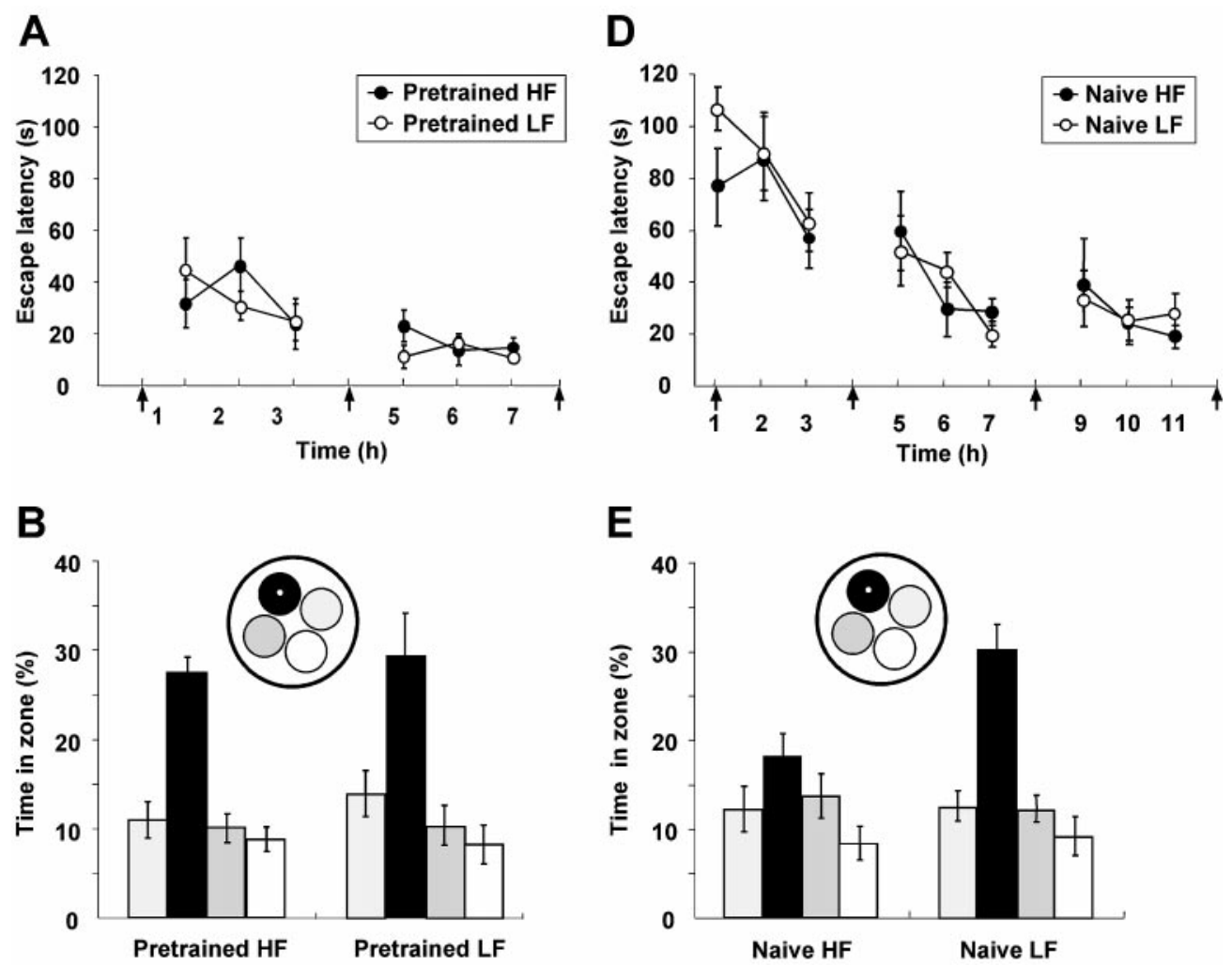

Figure 3. Effect of LTP saturation on learning a new water maze task in pretrained $(A-C)$ and naïve $(D-F)$ animals (means $\pm \mathrm{SEM}) . A, D$, Latency to enter the hidden platform during training after tetanic stimulation. Arrows indicate probe tests $B, E$, Performance on the final probe test, showing time inside a circle (64 $\mathrm{cm}$ diameter; inset) around the submerged platform (black) and in corresponding, equally large zones in the other pool quadrants. $C, F$, Development of spatial behavior (time in the platform zone) across probe tests, with zone time calculated as in $B$ and $E$. Probe test 1 was conducted before training started.
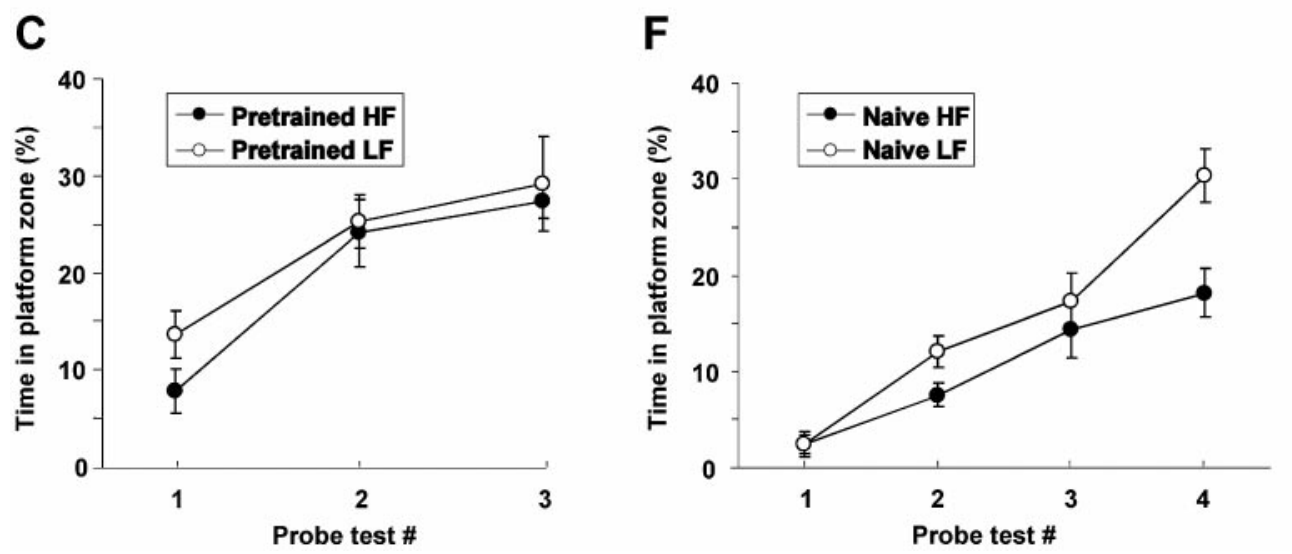

NMDA receptor antagonists display sensorimotor deficits (Saucier and Cain,1995; Cain et al., 1996), and the poor search behavior of naïve drug-treated rats may reflect inability to learn the basic motor strategies of the task rather than blockade of LTP. Pretraining may allow the necessary sensorimotor strategies to be acquired in advance (Cain, 1998). We show here that blockade of LTP by saturation impairs learning in naïve animals in the absence of sensorimotor impairment.

\section{Alternative interpretations}

It is unlikely that the failure to impair learning in pretrained $\mathrm{HF}$ rats reflects less successful saturation in this group than in the naïve HF group. Naïve and pretrained animals exhibited no differences in residual LTP. Learning was intact in all pretrained HF animals, even when no further LTP could be induced.

The pretrained groups received longer training than the naïve groups $(40+15$ trials vs 23 trials). Thus, the failure to block new learning in pretrained HF rats may be a consequence of a larger number of training sessions. Examination of performance on early trials in the second water maze suggests that this is not the case. The first probe test (at $4 \mathrm{hr}$ ) was conducted before robust learning had taken place in the pretrained animals, with performance being poorer than on the final probe test in the naïve groups. Nonetheless, although the naïve HF and LF groups were still different from each other at the end of training (12 hr), the pretrained groups had comparable search times already on the first probe test after training had started (Fig. $3 C$; probe test 2), suggesting that the pretraining effect was expressed from the outset.

However, even though the performance of the pretrained groups was quantitatively indistinguishable, the pretrained HF animals may have solved the second water maze task by using nonspatial, nonhippocampal strategies. When given extensive training, rats with bilateral hippocampal lesions are able to learn where a platform is located in a water maze (Morris et al., 1990; Whishaw et al., 1995). This slow type of learning may, at least partly, be nonspatial (Eichenbaum et al., 1990). It is unlikely that 
such learning was critical in the present experiment, because rats with complete hippocampal lesions failed to benefit from pretraining in a previous study with the same training protocol (Moser and Moser, 1998). Thus, the pretraining effect depends on the hippocampus.

\section{What is the LTP-dependent component of spatial learning?}

The pretrained animals benefited from the transfer of procedural knowlege from the first to the second water maze, such as knowing that there is an escape platform, swimming away from the pool walls, and using the platform as a refuge. Naïve animals may have been unable to acquire this type of knowledge when LTP was blocked. However, both of the naïve groups started out with high escape latencies that were significantly reduced during training, suggesting that, despite their apparently random search pattern on the probe tests, the naive HF rats had learned to search in the inner or middle area of the pool where they frequently encountered the platform by chance. This is consistent with a recent study reporting that rats profit from nonspatial pretraining even if NMDA receptors are blocked during pretraining (Hoh et al., 1999). Thus, significant procedural components of the water maze task do not seem to require NMDA receptor-dependent LTP.

Taken together, the studies of spatial learning after interference with LTP in pretrained animals suggest that hippocampal NMDA receptor-dependent LTP is not required for spatial learning per se or for learning the general strategies of the water maze task during pretraining. Nevertheless, animals are impaired when rats are required to learn both components of the task at once during blockade of further LTP formation. Hoh et al. (1999) have suggested that pretraining prevents the learning impairment by reducing task difficulty as the procedural and spatial components are learned separately. If "task difficulty" is the critical factor determining whether learning can take place in the absence of LTP, future studies must determine what makes a task "difficult"; i.e., which specific elements occur in conjunction only when animals learn procedural and spatial aspects of the task simultaneously.

One clue comes from the fact that pretraining does not help in a delayed-matching task. When rats are trained with a new platform position daily, NMDA receptor blockade blocks retention in a delay-dependent manner despite extensive drug-free pretraining (Steele and Morris, 1999). An important difference between this and other tasks is that target positions used on previous days must be ignored. Successful performance requires the animals to remember both where the platform was positioned and when the platform occupied this position (recent or remote). One function of LTP may be to associate such elements of experience (when and where) in episodic memory (Morris and Frey, 1997). Spatial learning may take place without LTP, but only when the other episodic aspects of the training context are familiar, as they are in the pretrained groups of the present experiment.

\section{REFERENCES}

Bannerman D, Good MA, Butcher SP, Ramsay M, Morris RGM (1995) Distinct components of spatial learning revealed by prior training and NMDA receptor blockade. Nature 378:182-186.

Bliss TVP, Collingridge GL (1993) A synaptic model of memory-longterm potentiation in the hippocampus. Nature 361:31-39.

Cain DP (1998) Testing the NMDA, long-term potentiation, and cholinergic hypotheses of spatial learning. Neurosci Biobehav Rev 22:181-193

Cain DP, Saucier D, Hall J, Hargreaves EL, Boon F (1996) Detailed behavioral analysis of water maze acquisition under APV or CNQX: contribution of sensorimotor disturbances to drug-induced acquisition deficits. Behav Neurosci 110:86-102.

Castro CA, Silbert LH, McNaughton BL, Barnes CA (1989) Recovery of spatial learning deficits after decay of electrically induced synaptic enhancement in the hippocampus. Nature 342:545-548.

Eichenbaum H, Stewart C, Morris RGM (1990) Hippocampal representation in place learning. J Neurosci 10:3531-3542.

Hoh T, Beiko J, Boon F, Weiss S, Cain DP (1999) Complex behavioral strategy and reversal learning in the water maze without NMDA receptor-dependent long-term potentiation. J Neurosci 19: RC2(1-5).

McNaughton BL, Barnes CA, Rao G, Baldwin J, Rasmussen M (1986) Long-term enhancement and the acquisition of spatial information. J Neurosci 6:563-571.

Morris RGM, Frey U (1997) Hippocampal synaptic plasticity: role in spatial learning or the automatic recording of attended experience? Philos Trans R Soc Lond B Biol Sci 352:1489-1503.

Morris RGM, Anderson E, Lynch GS, Baudry M (1986) Selective impairment of learning and blockade of long-term potentiation by an $N$-methyl-D-aspartate receptor antagonist, AP5. Nature 319:774-776.

Morris RGM, Schenk F, Tweedie F, Jarrard LE (1990) Ibotenate lesions of hippocampus and/or subiculum: dissociating components of allocentric spatial learning. Eur J Neurosci 2:1016-1028.

Moser EI, Mathiesen I, Andersen P (1993) Association between brain temperature and dentate field potentials in exploring and swimming rats. Science 259:1324-1326.

Moser EI, Krobert KA, Moser M-B, Morris RGM (1998) Impaired spatial learning after saturation of long-term potentiation. Science 281:2038-2042.

Moser M-B, Moser EI (1998) Distributed encoding and retrieval of spatial memory in the hippocampus. J Neurosci 18:7535-7542.

Sakimura K, Kutsuwada T, Ito I, Manabe T, Takayama C, Kushiya E, Yagi T, Aizawa S, Inoue Y, Sugiyama H, Mishina M (1995) Reduced hippocampal LTP and spatial learning in mice lacking NMDA receptor $\epsilon 1$ subunit. Nature 373:151-155.

Saucier D, Cain DP (1995) Spatial learning without NMDA receptordependent long-term potentiation. Nature 378:186-189.

Silva AJ, Paylor R, Wehner JM, Tonegawa S (1992) Impaired spatial learning in $\alpha$-calcium-calmodulin kinase II mutant mice. Science 257:206-211.

Steele RJ, Morris RGM (1999) Delay-dependent impairment of a matching-to-place task with chronic and intrahippocampal infusion of the NMDA-antagonist D-AP5. Hippocampus 9:118-136.

Tsien JZ, Huerta PT, Tonegawa S (1996) The essential role of hippocampal CA1 NMDA receptor-dependent synaptic plasticity in spatial memory. Cell 87:1327-1338.

Whishaw IQ, Cassel JC, Jarrard LE (1995) Rats with fimbria-fornix lesions display a place response in a swimming pool: a dissociation between getting there and knowing where. J Neurosci 15:5779-5788. 